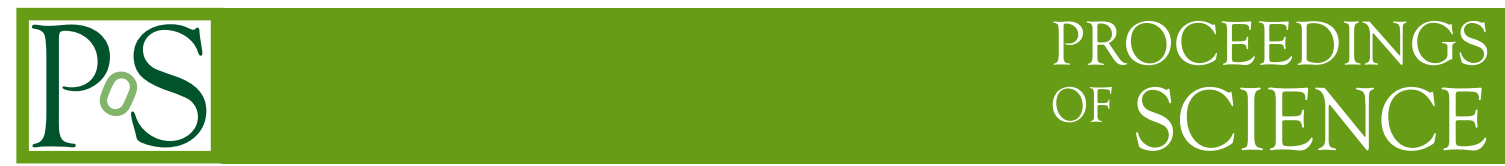

\title{
Highlights from BESIII
}

\author{
Yaqian Wang* \\ Johannes Gutenberg University \\ E-mail: whyaqmegmail.com
}

Starting from 2009, BESIII has collected large data samples of $J / \psi, \psi^{\prime}, \psi(3770), \psi(4040)$, $Y(4260)$, and $Y$ (4360) decays, which can be used for the study of $\tau$-charm physics. We present the current status of the machine and data analysis, including results from light hadron spectroscopy, charmonium spectroscopy, charm physics, and $X Y Z$ studies.

14th International Conference on B-Physics at Hadron Machines, April 8-12, 2013

Bologna, Italy

${ }^{*}$ Speaker. 


\section{Introduction}

BEPCII/BESIII [1] is an upgrade facility from the previous BEPC/BESII experiment. The BEPCII is a modern accelerator with multi-bunch, double ring and high luminosity. It can operate with the beam energy varying between 1.0 and $2.3 \mathrm{GeV}$. The design luminosity is $1 \times 10^{33} \mathrm{~cm}^{-2} \mathrm{~s}^{-1}$, and currently $65 \%$ of the target has been achieved. The BESIII spectrometer consists of a main drift chamber with momentum resolution of $0.5 \%$ at $1.0 \mathrm{GeV} / c$, an electromagnetic calorimeter with energy resolution $2.5 \%$ at $1.0 \mathrm{GeV}$, a Time-Of-Flight counter, a superconducting magnet with a magnetic strength of $1.0 \mathrm{~T}$, and a muon system made of resistive plate chambers.

With $2.25 \times 10^{8} \mathrm{~J} / \psi$ events, a partial wave analysis (PWA) is performed to search for glueballs, hybrids and multi-quark states [2]. Based on $1.06 \times 10^{8} \psi^{\prime}$ events, charmonium spectroscopy is widely studied [3]. The open charm physics is under study with $2.9 \mathrm{fb}^{-1}$ of data taken at the $\psi(3770)$ peak. Research on $X Y Z$ particles is performed with data taken at $\psi(4040), Y(4260)$, and $Y(4360)$ resonances.

\section{Light hadron physics}

According to lattice quantum chromodynamics (LQCD) calculations, the lowest mass glueball with $0^{++}$is in the mass region from 1.5 to $1.7 \mathrm{GeV} / \mathrm{c}^{2}$. Searching for glueballs at low energy is difficult due to its mixing with the nonet $q \bar{q}$ mesons. The two pseudoscalar meson final state in radiative $J / \psi$ decays is quite a clean laboratory to search for scalar and tensor glueballs.

\section{$2.1 \mathrm{~J} / \psi \rightarrow \gamma \eta \eta$}

The $f_{0}(1770)$ state was first observed in the $J / \psi \rightarrow \gamma \eta \eta$ decay mode by the Crystal Ball Collaboration [4]. To improve the statistical limit, we studied the decay $J / \psi \rightarrow \gamma \eta \eta, \eta \rightarrow \gamma \gamma$ [5] with $2.25 \times 10^{8} \mathrm{~J} / \psi$ events. The basic solution from PWA includes contribution from $f_{0}(1500)$, $f_{0}(1710), f_{0}(2100), f_{2}^{\prime}(1525), f_{2}(1810)$, and $f_{2}(2340)$. The dominant scalar components are $f_{0}(1710)$ and $f_{0}(2100)$, which are almost one order larger than $f_{0}(1500)$. The tensor components are dominantly $f_{2}(1525), f_{2}(1810)$, and $f_{2}(2340)$. The significant contribution from $f_{2}(1525)$ is shown as a clear peak in the mass spectrum of $\eta \eta$. A tensor component exists in the mass region between 1.8 and $2.0 \mathrm{GeV} / c^{2}$, although we can not distinguish $f_{2}(1810)$ from $f_{2}(1910)$ or $f_{2}(1950)$. The PWA requires a strong contribution from $f_{2}(2340)$, although the possibility of $f_{2}(2300)$ cannot be ruled out. The measured masses, widths, and branching fractions are listed in Table 1.

\section{$2.2 \mathrm{~J} / \psi \rightarrow \gamma \omega \phi$}

The $J / \psi \rightarrow \gamma \omega \phi$ decay mode is a doubly OZI suppressed process with a production rate that is expected to be suppressed relative to $J / \psi \rightarrow \gamma \omega \omega$ and $J / \psi \rightarrow \gamma \phi \phi$ by at least one order of magnitude [6]. The decay mode $J / \psi \rightarrow \gamma \omega \phi\left(\omega \rightarrow \pi^{+} \pi^{-} \pi^{0}, \phi \rightarrow K^{+} K^{-}\right)$is studied by means of data sample composed of $2.25 \times 10^{8} \mathrm{~J} / \psi$ mesons. A PWA with a tensor covariant amplitude is performed assuming that the enhancement is due to the presence of a resonance, the $X(1810)$, and confirms that the spin-parity of the $X(1810)$ is $0^{++}$[7]. The PWA results are summarised in Table 2, and are consistent within errors with those of the BESII experiment [8]. 
Table 1: Summary of the PWA results, including masses and widths for resonances, branching fractions of $J / \psi \rightarrow \gamma \mathrm{X}$, as well as significances. The first errors are statistical and the second systematic.

\begin{tabular}{ccccc}
\hline \hline Resonance & Mass $\left(\mathrm{MeV} / c^{2}\right)$ & Width $\left(\mathrm{MeV} / c^{2}\right)$ & $\mathscr{B}(J / \psi \rightarrow \gamma X \rightarrow \gamma \eta \eta)$ & Significance \\
\hline$f_{0}(1500)$ & $1468_{-15-74}^{+14+23}$ & $136_{-26-100}^{+41+28}$ & $\left(1.65_{-0.31-1.40}^{+0.26+0.51}\right) \times 10^{-5}$ & $8.2 \sigma$ \\
$f_{0}(1710)$ & $1759 \pm 6_{-25}^{+14}$ & $172 \pm 10_{-16}^{+32}$ & $\left(2.35_{-0.111-0.74}^{+0.13+14}\right) \times 10^{-4}$ & $25.0 \sigma$ \\
$f_{0}(2100)$ & $2081 \pm 13_{-36}^{+24}$ & $273_{-24-23}^{+27+70}$ & $\left(1.13_{-0.010-0.28}^{+0.09}\right) \times 10^{-4}$ & $13.9 \sigma$ \\
$f_{2}^{\prime}(1525)$ & $1513 \pm 5_{-10}^{+4}$ & $75_{-10-8}^{+12+16}$ & $\left(3.42_{-0.51-1.30}^{+0.43}\right) \times 10^{-5}$ & $11.0 \sigma$ \\
$f_{2}(1810)$ & $1822_{-24-57}^{+29+66}$ & $229_{-42-155}^{+52+88}$ & $\left(5.40_{-0.67-2.35}^{+0.60+3.42}\right) \times 10^{-5}$ & $6.4 \sigma$ \\
$f_{2}(2340)$ & $2362_{-30-63}^{+31+140}$ & $334_{-54-100}^{+62+165}$ & $\left(5.60_{-0.65-2.07}^{+0.62 .37}\right) \times 10^{-5}$ & $7.6 \sigma$ \\
\hline \hline
\end{tabular}

Table 2: Results from the best PWA fit solution.

\begin{tabular}{cccccccc}
\hline \hline Resonance & $\mathrm{J}^{P C}$ & $\mathrm{M}\left(\mathrm{MeV} / c^{2}\right)$ & $\Gamma\left(\mathrm{MeV} / c^{2}\right)$ & Events & $\Delta \mathscr{S}$ & $\Delta$ ndf & Significance \\
\hline$X(1810)$ & $0^{++}$ & $1795 \pm 7$ & $95 \pm 10$ & $1319 \pm 52$ & 783 & 4 & $>30 \sigma$ \\
\hline $\mathrm{f}_{2}(1950)$ & $2^{++}$ & 1944 & 472 & $665 \pm 40$ & 211 & 2 & $20.4 \sigma$ \\
\hline $\mathrm{f}_{0}(2020)$ & $0^{++}$ & 1992 & 442 & $715 \pm 45$ & 100 & 2 & $13.9 \sigma$ \\
\hline$\eta(2225)$ & $0^{-+}$ & 2226 & 185 & $70 \pm 30$ & 23 & 2 & $6.4 \sigma$ \\
\hline phase space & $0^{-+}$ & - & - & $319 \pm 24$ & 45 & 2 & $9.1 \sigma$ \\
\hline \hline
\end{tabular}

The anomalous enhancement observed at the $\omega \phi$ invariant-mass threshold and the large measured branching fractions (about half of $\mathscr{B}(J / \psi \rightarrow \gamma \phi \phi)$ [9]) are surprising and interesting. The enhancement is not compatible with being due either to the $X(1835)$ or the $X(p \bar{p})$, due to the different mass and spin-parity. The interpretation of the enhancement as being due to effects of $\omega \phi$ final state interactions (FSI) is not excluded in this analysis. Searches for this structure in different decays modes, e.g. $K^{*} K^{*}, \omega \omega$, etc., and in other production processes, e.g. $J / \psi \rightarrow \phi \omega \phi$, $J / \psi \rightarrow \omega \omega \phi$, etc., are essential to explore the nature of the enhancement, and gain more insight in the underlying dynamics.

\section{Charmonium spectroscopy}

\subsection{Mass and width of $\eta_{c}$}

Properties of $\eta_{c}$ are not well understood, although this state has been observed for many years. There are obvious discrepancies between results from different experiments [10]. An obvious distortion in the line shape of the $\eta_{c}$ is reported by CLEO [11], but similar effects are not observed in $\psi^{\prime} \rightarrow \pi^{0} h_{c}, h_{c} \rightarrow \gamma \eta_{c}$ at BESIII.

Measurements of $\eta_{c}$ at BESIII are performed with six decay channels, including $K_{S}^{0} K \pi$, $K^{+} K^{-} \pi^{0}, \pi^{+} \pi^{-} \eta, K_{S}^{0} K 3 \pi, K^{+} K^{-} \pi^{+} \pi^{-} \pi^{0}$ and $3\left(\pi^{+} \pi^{-}\right)$[12]. A simultaneous fit with unique $\eta_{c}$ mass and width is performed on the $\eta_{c}$ mass spectra, where a full interference between $\eta_{c}$ and non-resonant $\psi^{\prime}$ radiative decays is considered, and the quantum numbers of the non- $\eta_{c}$ components are assumed to be $0^{-+}$. The corresponding relative phases in different decay modes are found to be consistent within $3 \sigma$, which are constrained to the same value in the final fit. The obtained results are $M_{\eta_{c}}=2984.3 \pm 0.6 \pm 0.6 \mathrm{MeV} / c^{2}, \Gamma_{\eta_{c}}=32.0 \pm 1.2 \pm 1.0 \mathrm{MeV}, \phi_{1}=2.40 \pm 0.07 \pm 0.08$ 
$\operatorname{rad}$ (constructive), and $\phi_{2}=4.19 \pm 0.03 \pm 0.09 \mathrm{rad}$ (destructive). The BESIII results are consistent with those from two-photon production $[13,14,15]$. The precisions of the measured mass and width are improved.

\subsection{First observation of $\psi^{\prime} \rightarrow \gamma \eta_{c}^{\prime}$}

The $\eta_{c}^{\prime}$ state was first observed by the Belle Collaboration [16] in $B \rightarrow K K_{S} K^{-} \pi^{+}$decay, and is also expected in the radiative transition of $\psi^{\prime}$. According to the potential model, the branching fraction is predicted to be $\mathscr{B}\left(\psi^{\prime} \rightarrow \gamma \eta_{c}^{\prime}\right)=(0.1-6.2) \times 10^{-4}$ [17].

BESIII performed a search for the $\eta_{c}^{\prime}$ in several decay modes, and a signal is only observed in the $K \bar{K} \pi$ [18] final state. Fig. 1 shows the fit result to the mass spectrum of $K \bar{K} \pi$. With a
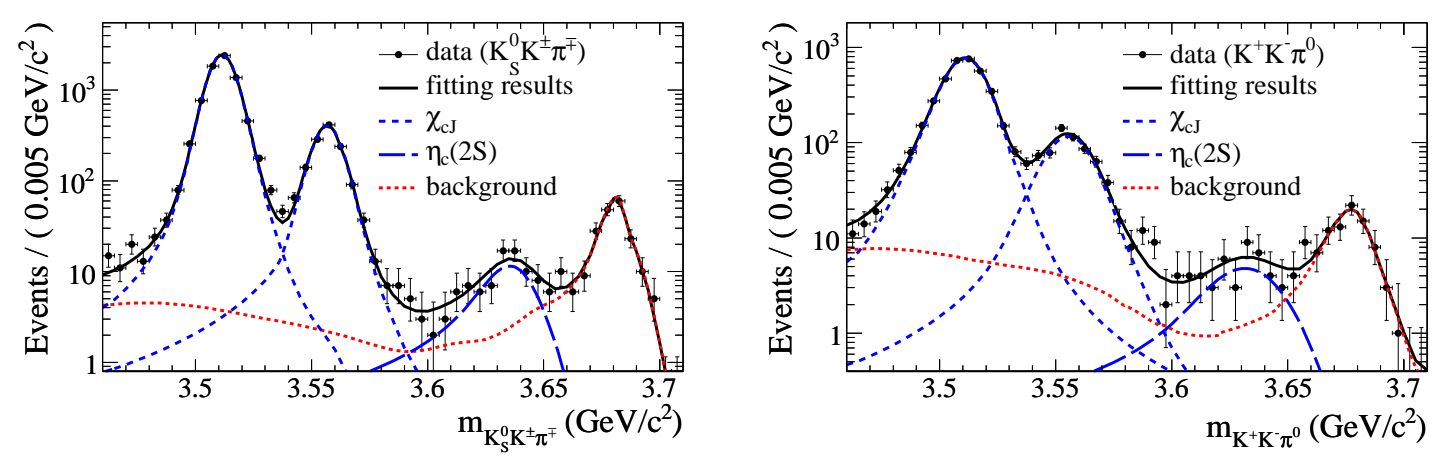

Figure 1: The simultaneous fit to the $K \bar{K} \pi$ mass spectrum.

simultaneous fit we determine the mass and width of the $\eta_{c}^{\prime}$ to be: $M_{\eta_{c}^{\prime}}=3637.6 \pm 2.9 \pm 1.6$ $\mathrm{MeV} / c^{2}$, and $\Gamma_{\eta_{c}^{\prime}}=16.9 \pm 6.4 \pm 4.8 \mathrm{MeV}$. The visible branching fraction is determined to be $\mathscr{B}\left(\psi^{\prime} \rightarrow \gamma \eta_{c}^{\prime}\right) \times \mathscr{B}\left(\eta_{c}^{\prime} \rightarrow K \bar{K} \pi\right)=(1.30 \pm 0.20 \pm 0.30) \times 10^{-5}$. Using the previous measurement and $\mathscr{B}\left(\eta_{c}^{\prime} \rightarrow K \bar{K} \pi\right)=(1.9 \pm 0.4 \pm 1.1) \%$ from BaBar [19], the M1 transition rate is determined to be $\mathscr{B}\left(\psi^{\prime} \rightarrow \gamma \eta_{c}^{\prime}\right)=(6.8 \pm 1.1 \pm 4.5) \times 10^{-4}$.

\section{Charm physics}

\section{1 $D^{+} \rightarrow \mu^{+} v_{\mu}$ (preliminary)}

The decay constant $f_{D^{+}}$is an important constant in heavy flavor physics. Within the context of the standard model, the measurement of the purely leptonic decay of the $\mathrm{D}$ meson provides a means for determining $f_{D}^{+}$. With the precisely measured branching fraction of the $D^{+} \rightarrow \mu^{+} v_{\mu}$ decay together with the accurate calculation of $f_{D}^{+}$from unquenched LQCD, we can extract $\left|V_{c d}\right|$ more precisely. Charged $D$ mesons are produced in $\psi(3770) \rightarrow D^{+} D^{-}$decays. The analysis is based on the $D$-tag technique. On the tagged side, $D^{-}$is reconstructed from nine hadronic modes, including $K^{+} \pi^{-} \pi^{-}, K_{S}^{0} \pi^{-}, K_{S}^{0} K^{-}, K^{+} K^{-} \pi^{-}, K^{+} \pi^{-} \pi^{-} \pi^{0}, \pi^{+} \pi^{-} \pi^{-}, K_{S}^{0} \pi^{-} \pi^{0}, K^{+} \pi^{-} \pi^{-} \pi^{-} \pi^{+}$, and $K_{S}^{0} \pi^{-} \pi^{-} \pi^{+}$. The $D^{+} \rightarrow \mu^{+} v_{\mu}$ signal is obtained from the fit to the $M_{\text {miss }}=E_{\text {miss }}-P_{\text {miss }}$ distribution. The signal yield is $377.3 \pm 20.6 \pm 2.6$ events, and the obtained branching fraction 
$\mathscr{B}\left(D^{+} \rightarrow \mu^{+} v_{\mu}\right)=(3.74 \pm 0.21 \pm 0.06) \times 10^{-4}$, which is the world best measurement. The error is still limited by the statistics.

\section{2 $D^{0} \rightarrow K^{+} e^{-} v_{e}$ and $D^{0} \rightarrow \pi^{+} e^{-} v_{e}$ (preliminary)}

Semileptonic decays of $D$ mesons are an excellent environment for precision measurements of the Cabibbo-Kobayashi-Maskawa (CKM) matrix elements. However, to determine the CKM weak parameters, knowledge of strong interaction effects is required. These effects can be parametrized by form factors. Techniques such as LQCD offer increasingly precise calculations of these form factors. As the uncertainties in the predictions shrink, experimental validation of the results becomes more important. The $D^{0}$ mesons are produced from $\psi(3770) \rightarrow D^{0} \bar{D}^{0}$ decays. The tagged$D^{0}$ is reconstructed from four hadronic modes. The amount of signal events is determined by fitting the distribution of $U_{\text {miss }}=E_{\text {miss }}-\left|\vec{p}_{\text {miss }}\right|$. Based on $0.9 \mathrm{fb}^{-1}$ of data taken at the $\psi(3770)$ peak, preliminary results on the branching fractions are measured to be: $\mathscr{B}\left(\bar{D}^{0} \rightarrow K^{+} e^{-} \bar{v}\right)=$ $(3.542 \pm 0.030 \pm 0.067) \times 10^{-2}$ and $\mathscr{B}\left(\bar{D}^{0} \rightarrow \pi^{+} e^{-} \bar{v}\right)=(0.288 \pm 0.008 \pm 0.005) \times 10^{-2}$.

\section{5. $X Y Z$ studies}

\subsection{First observation of $e^{+} e^{-} \rightarrow \eta J / \psi$ at $\sqrt{s}=4.009 \mathrm{GeV}$}

BESIII observed for the first time the production of $e^{+} e^{-} \rightarrow \eta J / \psi[20]$ at center-of- mass energy $\sqrt{s}=4.009 \mathrm{GeV}$ with a statistical significance greater than $10 \sigma$. In this analysis, the $J / \psi$ is reconstructed through its decays into lepton pairs $\left(e^{+} e^{-}\right.$and $\left.\mu^{+} \mu^{-}\right)$, while $\eta$ is reconstructed with the $\gamma \gamma$ final state. The Born cross section is measured to be $(32.1 \pm 2.8 \pm 1.3) \mathrm{pb}$. Assuming that the $\eta J / \psi$ signal is purely from hadronic decays of the $\psi(4040)$, the fractional transition rate is determined to be $\mathscr{B}(\psi(4040) \rightarrow \eta J / \psi)=(5.2 \pm 0.5 \pm 0.2 \pm 0.5) \times 10^{-3}$, where the third error is the uncertainty from $\psi(4040)$ resonance parameters.

\subsection{Observation of $Z_{c}(3900)$}

The $Y(4260)$ state was discovered in the initial-state-radiation (ISR) process $e^{+} e^{-} \rightarrow \gamma_{\text {ISR }} \pi^{+} \pi^{-}$ $J / \psi[21]$. Unlike other charmonium states with the same quantum numbers and in the same mass region, such as the $\psi(4040), \psi(4160)$, and $\psi(4415)$, the $Y(4260)$ state does not have a natural place within the quark model of charmonium [22]. Furthermore, while being well above the $D \bar{D}$ threshold, the $Y$ (4260) shows strong coupling to the $\pi^{+} \pi^{-} J / \psi$ final state [23], but relatively small coupling to open charm decay modes $[24,25,26,27,28]$. In this talk, we present a study of the process $e^{+} e^{-} \rightarrow \pi^{+} \pi^{-} J / \psi$ [29] at a center-of-mass energy of $\sqrt{s}=(4.260 \pm 0.001) \mathrm{GeV}$, which corresponds to the peak of the $Y(4260)$ cross section. The cross section is measured to be $(62.9 \pm 1.9 \pm 3.7) \mathrm{pb}$, which agrees with the existing results from the BaBar [30], Belle [31], and CLEO [32] experiments. In addition, a structure with a mass of $(3899.0 \pm 3.6 \pm 4.9) \mathrm{MeV} / c^{2}$ and a width of $(46 \pm 10 \pm 20) \mathrm{MeV}$ is observed in the $\pi^{+} \pi^{-} J / \psi$ mass spectrum as shown in Fig. 2. This structure couples to charmonium and has an electric charge, i.e. due to a state containing more quarks than just a charm and anti-charm quark. Similar studies were performed in $B$ decays, with unconfirmed structures reported in the $\pi^{+} \pi^{-} \psi^{\prime}$ and $\pi^{ \pm} \chi_{c 1}$ systems [33, 34, 35, 36]. Model-dependent calculations that attempt to explain the charged bottomonium-like structures and 


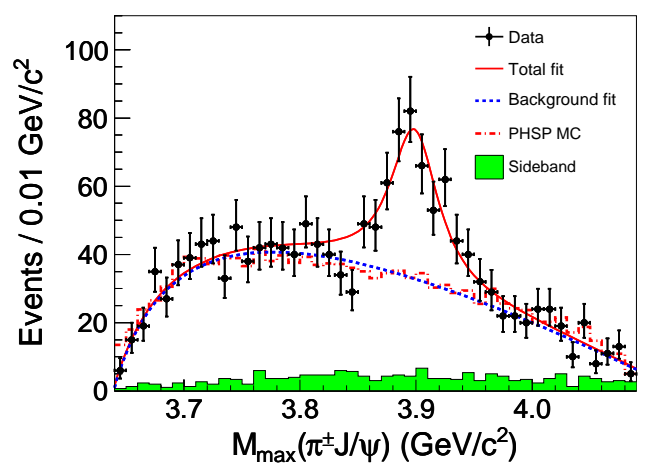

Figure 2: Fit to the $M_{\max }\left(\pi^{ \pm} J / \psi\right)$ distribution. Dots with error bars are data; the red solid curve shows the total fit, and the blue dotted curve the background from the fit; the red dot-dashed histogram shows the result of a phase space MC simulation; and the green shaded histogram shows the normalized $J / \psi$ sideband events.

may also apply to the charmonium-like structures exist. There are predictions of charmonium-like structures near the $D \bar{D}^{*}$ and $D^{*} \bar{D}^{*}$ thresholds [37].

\section{Summary}

We have shown the recent results from the BESIII Collaboration, including analyses from light hadron spectroscopy, charmonium spectroscopy, charm physics, as well as new results on the $X Y Z$ states. With the excellent performance of the accelerator and the detector, more interesting results are expected to come soon.

\section{References}

[1] M. Ablikim et al. (BESIII Collaboration), Nucl. Instrum. Methods Phys. Res., Sect. A 614, 345 (2010).

[2] M. Ablikim et al. (BESIII Collaboration), Chinese Phsyics C 36 (10), 915-925 (2012).

[3] M. Ablikim et al. (BESIII Collaboration), arXiv:1209.6199 [hep-ph].

[4] C. Edwards et al., Phys. Rev. Lett. 48, 458 (1982).

[5] M. Ablikim et al. (BESIII Collaboration), Phys. Rev. D. 87, 092009 (2013).

[6] Köpke L, Wermes N. J/ $\psi$ Decays. CERN-EP/88-93, Physics Reports 174 (1989) 67-227: CERN, CH-1211 Geneva 23, Switzerland.

[7] M. Ablikim et al. (BESIII Collaboration), Phys. Rev. D 87, 032008 (2013).

[8] M. Ablikim et al. (BES Collaboration), Phys. Rev. Lett. 96, 162002 (2006).

[9] J. Beringer et al. (Particle Data Group), Phys. Rev. D 86, 010001 (2012).

[10] K. Nakamura et al., Journal of Physics G 37, 075021 (2010).

[11] R.E. Mitchell et al. (CLEO Collaboration), Phys. Rev. Lett. 102, 011801 (2009). 
[12] M. Ablikim et al. (BESIII Collaboration), Phys. Rev. Lett. 108, 222002 (2012).

[13] D. M. Asner et al. (CLEO Collaboration), Phys. Rev. Lett. 92, 142001 (2004).

[14] B. Aubert et al. (BABAR Collaboration), Phys. Rev. Lett. 92, 142002 (2004).

[15] S. Uehara et al. (Belle Collaboration), Eur. Phys. J. C 53, 1 (2008).

[16] S.K. Choi et al. (Belle Collaboration), Phys. Rev. Lett. 89, 102001 (2002).

[17] K. Gao, PhD thesis, arXiv: 0909.2812.

[18] M. Ablikim et al. (BESIII Collaboration), Phys. Rev. Lett. 109, 042003 (2012).

[19] B. Aubert et al. (BABAR Collaboration), Phys. Rev. D 78, 012006 (2008).

[20] M. Ablikim et al. (BESIII Collaboration), Phys. Rev. D 86, 071101 (2012).

[21] B. Aubert et al. (BaBar Collaboration), Phys. Rev. Lett. 95, 142001 (2005).

[22] X. H. Mo et al., Phys. Lett. B 640, 182 (2006).

[23] D. Cronin-Hennessy et al. (CLEO Collaboration), Phys. Rev. D 80, 072001 (2009).

[24] G. Pakhlova et al. (Belle Collaboration), Phys. Rev. Lett. 98, 092001 (2007).

[25] B. Aubert et al. (BaBar Collaboration), Phys. Rev. D 76, 111105 (2007).

[26] B. Aubert et al. (BaBar Collaboration), Phys. Rev. D 79, 092001 (2009).

[27] P. del Amo Sanchez et al. (BaBar Collaboration), Phys. Rev. D 82, 052004 (2010).

[28] For a recent review, see N. Brambilla et al., Eur. Phys. J. C 71, 1534 (2011).

[29] M. Ablikim et al. (BESIII Collaboration), arXiv:1303.5949 [hep-ph].

[30] J. P. Lees et al. (BaBar Collaboration), Phys. Rev. D 86, 051102(R) (2012).

[31] C. Z. Yuan et al. (Belle Collaboration), Phys. Rev. Lett. 99, 182004 (2007).

[32] T. E. Coan et al. (CLEO Collaboration), Phys. Rev. Lett. 96, 162003 (2006).

[33] S. K. Choi et al. (Belle Collaboration), Phys. Rev. Lett. 100, 142001 (2008).

[34] B. Aubert et al. (BaBar Collaboration), Phys. Rev. D 79, 112001 (2009).

[35] R. Mizuk et al. (Belle Collaboration), Phys. Rev. D 78, 072004 (2008).

[36] J. P. Lees et al. (BaBar Collaboration), Phys. Rev. D 85, 052003 (2012).

[37] L. Maiani, F. Piccinini, A. D. Polosa and V. Riquer, Phys. Rev. D 71, 014028 (2005); D. -Y. Chen, X. Liu and T. Matsuki, arXiv:1208.2411 [hep-ph]; D. -Y. Chen and X. Liu, Phys. Rev. D 84, 034032 (2011); Z. -F. Sun, J. He, X. Liu, Z. -G. Luo and S. -L. Zhu, Phys. Rev. D 84, 054002 (2011) and Chin. Phys. C 36, 194 (2012); Ahmed Ali, Christian Hambrock and Wei Wang, Phys. Rev. D 85, 054011 (2012). 